\title{
IMPROVEMENT OF COMPLEXITY AND PERFORMANCE OF LEAST SQUARE BASED CHANNEL ESTIMATION IN MIMO SYSTEM
}

\author{
Anand Kumar Sah ${ }^{1}$, Arun Kumar Timalsina ${ }^{2}$ \\ ${ }^{1}$ Department of Electronics and Computer Engineering \\ Western Region Campus, Institute of Engineering, Tribhuvan University \\ Lamachaur, Pokhara, Nepal \\ ${ }^{2}$ Department of Electronics and Computer Engineering \\ Central Campus, Institute of Engineering, Tribhuvan University \\ Pulchowk, Nepal
}

\begin{abstract}
Multiple-input multiple-output (MIMO) systems play a vital role in fourth generation wireless systems to provide advanced data rate. In this paper, a better performance and reduced complexity channel estimation method is proposed for MIMO systems based on matrix factorization. This technique is applied on training based least squares (LS) channel estimation using STBC for performance improvement. Simulation results indicate that the proposed method not only alleviates the performance of MIMO channel estimation but also significantly reduces the complexity caused by matrix inversion. The performance evaluations are validated through computer simulations using MATLAB in terms of bit error rate (BER) for modified LS with LS and MMSE channel estimation techniques. Simulation results show that the BER performance and complexity of the proposed method clearly outperforms the conventional LS channel estimation method.
\end{abstract}

Keywords: MIMO, STBC, LS, MMSE, Matrix Factorization

\section{Introduction}

Wireless communication systems continue to strive for ever higher data rates. To cater to both higher transmission rates and higher spectral efficiencies in order to increase the performance of communication systems, the wireless industry is already looking ahead and embracing multiple-input multiple-output (MIMO) systems [1,2]. Using multiple transmit as well as receive antennas, a MIMO system exploits spatial diversity, higher data rate, greater coverage and improved link robustness without increasing total transmission power or bandwidth. However, MIMO relies upon the knowledge of channel state information (CSI) at the receiver for data detection and decoding. It has been proved that when the channel is Rayleigh fading and perfectly known to the receiver, the performance of a MIMO system grows linearly with the number of transmit or receive antennas, whichever is less [3]. Therefore, an accurate and robust estimation of wireless channel is of crucial importance for coherent demodulation in MIMO system.

A considerable number of channel estimation methods have already been studied by different researchers for MIMO systems. In certain channel estimation methods, training symbols that are transmitted over the channels are investigated at the receiver to render accurate CSI [4]. Compared with blind and semi blind channel estimations, training based estimations generally require a small data record. Hence, they are not limited to slowly time-varying channels and entail less complexity. One of the most efficient training based methods is the 
least squares (LS) method, for which the channel coefficients are treated as deterministic but unknown constants [5]. When the full or partial information of the channel correlation is known, a better channel estimation can be achieved by minimum mean square error (MMSE) method [5]. The fundamental difference between these two techniques is that the channel coefficients are treated as deterministic but unknown constants in the former, and as random variables of a stochastic process in the latter. The MMSE estimation has better performance than LS estimation at the cost of higher complexity as it additionally exploits prior knowledge of the channel coefficients. But practically this kind of information is sometimes not known beforehand, which makes MMSE-based technique infeasible.

OFDM is becoming widely applied in wireless communications systems due to its high rate transmission capability with high bandwidth efficiency and its robustness with regard to multipath fading and delay [1]. It has been used in digital audio broadcasting (DAB) systems, digital video broadcasting (DVB) systems, digital subscriber line (DSL) standards, and wireless LAN standards such as the American IEEE® Std. 802.11 ${ }^{\mathrm{TM}}$ (WiFi) and its European equivalent HIPRLAN/2. It has also been proposed for wireless broadband access standards such as IEEE Std. 802.16 ${ }^{\mathrm{TM}}$ (WiMAX) and as the core technique for the fourth-generation $(4 \mathrm{G})$ wireless mobile communications.

There are two main problems in designing channel estimators for wireless OFDM systems. The first problem is the arrangement of pilot information, where pilot means the reference signal used by both transmitters and receivers. The second problem is the design of an estimator with both low complexity and good channel tracking ability. The two problems are interconnected. In general, the fading channel of OFDM systems can be viewed as a twodimensional (2D) signal (time and frequency). The optimal channel estimator in terms of mean-square error is based on 2D Wiener filter interpolation. Unfortunately, such a 2D estimator structure is too complex for practical implementation. The combination of high data rates and low bit error rates in OFDM systems necessitates the use of estimators that have both low complexity and high accuracy, where the two constraints work against each other and a good trade-off is needed. The one-dimensional (1D) channel estimations are usually adopted in OFDM systems to accomplish the trade-off between complexity and accuracy [14]. The two basic 1D channel estimations are block-type pilot channel estimation and combtype pilot channel estimation, in which the pilots are inserted in the frequency direction and in the time direction, respectively. The estimations for the block-type pilot arrangement can be based on least square (LS), minimum mean-square error (MMSE), and modified MMSE.

\section{Problem Definition}

The complexity of channel estimation mainly increases due to matrix inversion. To reduce the complexity of MIMO detection, several matrix factorization techniques have been applied on MIMO systems recently. Orthogonal matrix triangularization is a matrix factorization technique that reduces a full rank matrix into simpler form. Some other matrix factorizations like lower upper (LU) decomposition, singular value decomposition (SVD) can also be used to avoid explicit matrix inversions. But orthogonal matrix triangularization is preferable over other methods as it guarantees numerical stability by minimizing errors caused by machine roundoffs [6]. Matrix factorization is used to conduct large matrix calculations in alternate ways, and applied for system complexity reduction. The decoding algorithm for layered space-time codes based on matrix triangularization is 
presented in [7]. The authors in [8] proposed a low-complexity maximum-likelihood decoding approach based on matrix factorization for signal detection in MIMO systems. In [9], a combined detection algorithm based on matrix triangularization is proposed to reduce the complexity of the MIMO detection algorithm. Reduced complexity hardware architecture for MIMO symbol detector using matrix factorization is proposed in [13] which support two MIMO schemes of space-frequency block codes and space division multiplexing of the codes. However, in all works matrix factorizations decrease complexity, but performance improvement is not justified. In this paper, a channel estimation method is proposed for MIMO system by employing orthogonal matrix triangularization on LS estimation which minimizes the computational complexity and at the same time improves the performance. The coding scheme of MIMO considered in this paper is space-time block coding (STBC) which is an attractive approach for improving quality in wireless links.

\section{MIMO System and Model}

Digital communication using multiple-input-multiple-output (MIMO), sometimes called a "volumeto-volume" wireless link, has recently emerged as one of the most significant technical breakthroughs in modern communications. MIMO systems can be defined simply. Given an arbitrary wireless communication system, we consider a link for which the transmitting end as well as the receiving end is equipped with multiple antenna elements. The idea behind MIMO is that the signals on the transmit (TX) antennas at one end and the receive (RX) antennas at the other end are "combined" in such a way that the quality or the data rate (bits/sec) of the communication for each MIMO user will be improved. Such an advantage can be used to increase both the network's quality of service and the operator's revenues significantly. A compressed digital source in the form of a binary data stream is fed to a simplified transmitting block encompassing the functions of error control coding and (possibly joined with) mapping to complex modulation symbols. The latter produces several separate symbol streams. Each is then mapped onto one of the multiple TX antennas. After upward frequency conversion, filtering and amplification, the signals are launched into the wireless channel. At the receiver, the signals are captured by possibly multiple antennas and demodulation and de-mapping operations are performed to recover the message.

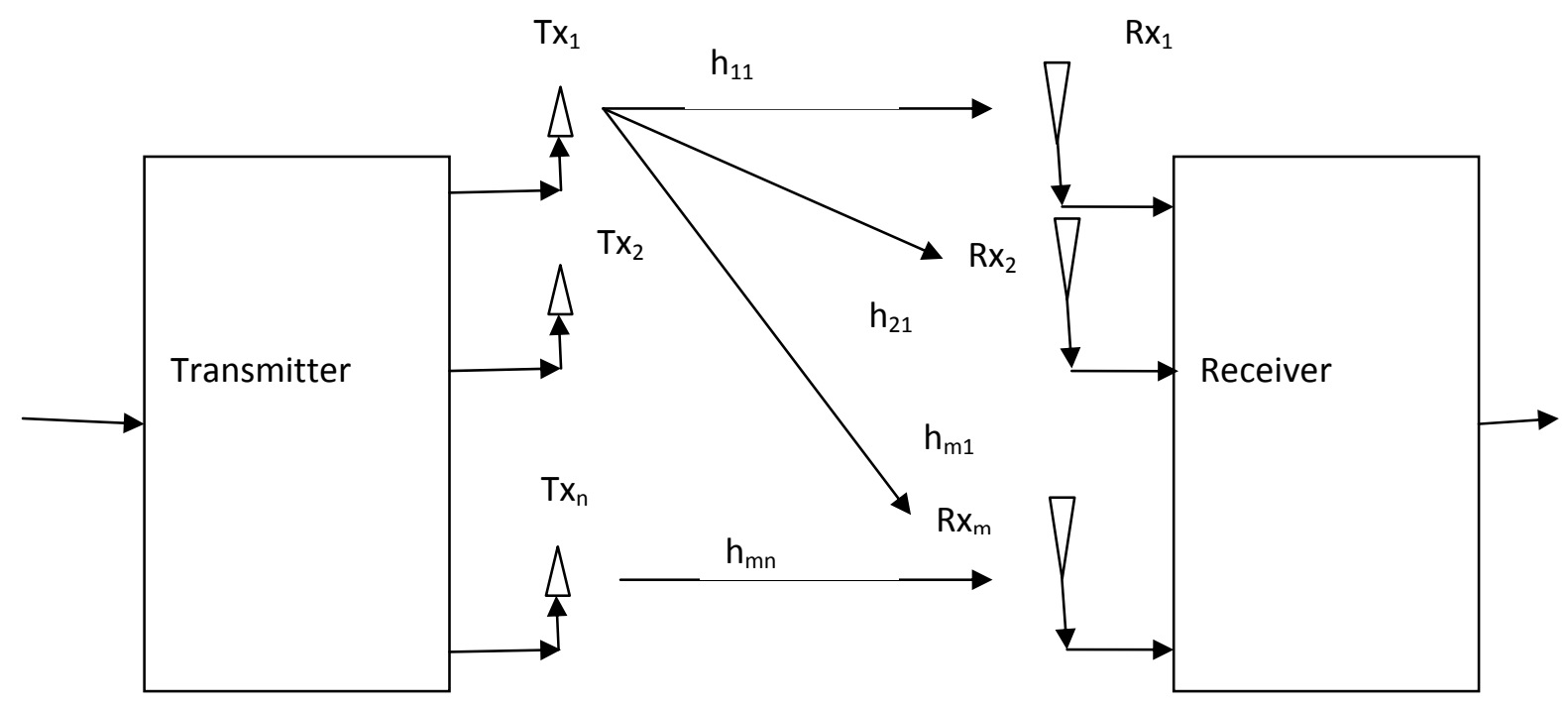

Fig 3.1 MIMO system with $\mathrm{N}$ transmit and $\mathrm{M}$ receive antenna 
In a single user MIMO model with $\mathrm{N}$ transmit and $\mathrm{M}$ receive antennas, the MIMO system equation is given by

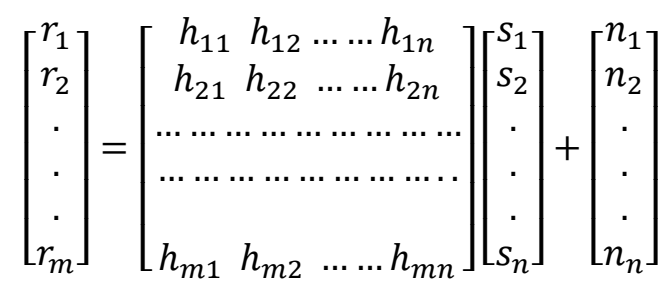

Or in matrix form: $\quad \mathrm{r}=\mathrm{Hs}+\mathrm{n}$

where $\mathrm{H}$ is the channel matrix of size $\mathrm{M} \times \mathrm{N}, \mathrm{r}$ is the $\mathrm{M} \times 1$ received signal vector, $\mathrm{s}$ is the $\mathrm{N} \times 1$ transmitted signal vector, and $\mathrm{n}$ is an $\mathrm{Mx} 1$ additive white Gaussian noise vector with zero mean and variance $\sigma^{2}$. The channel matrix $H$ is the factor by which the signal is amplified and is also known as the channel coefficient. The element $h_{i j}$ in the channel matrix $H$ represents the complex gain between transmitter antenna $\mathrm{j}$ and receiver antenna $\mathrm{i}$.

The MIMO system model is shown in Fig 3.2.

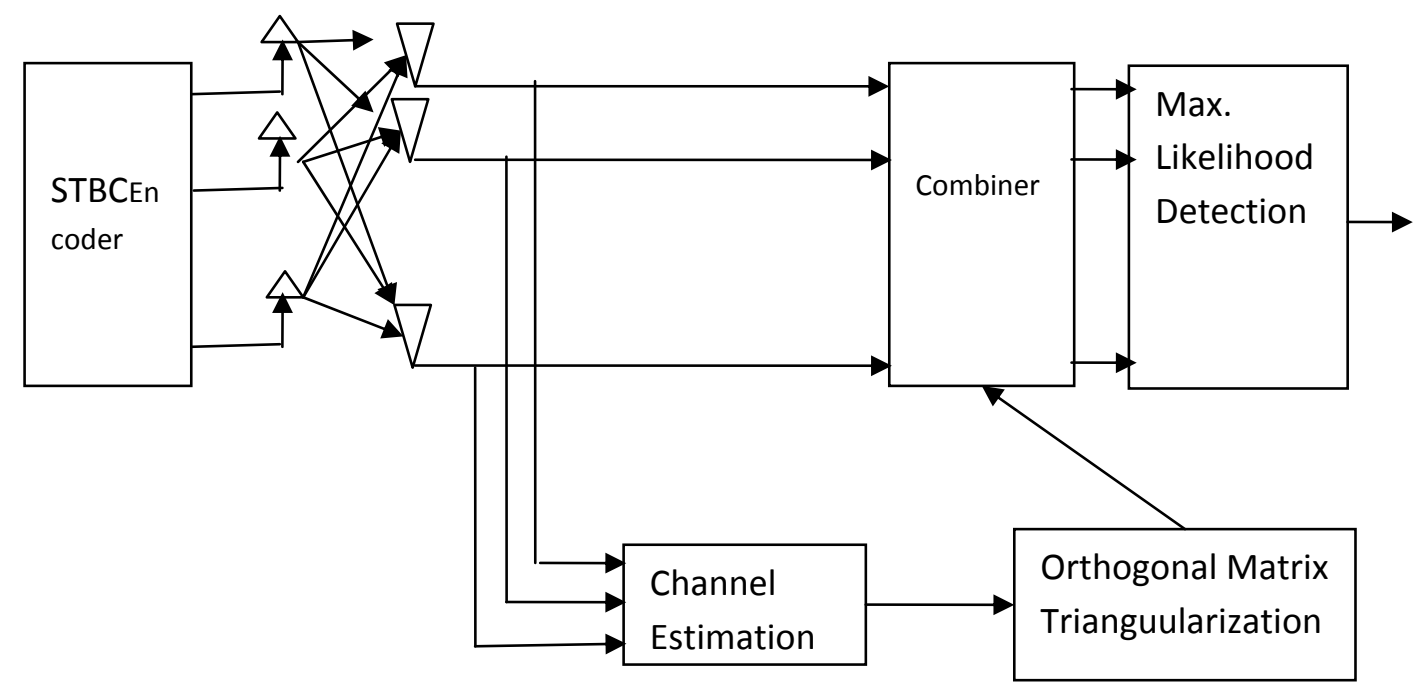

Fig 3.2 MIMO System Model

Data symbols $\mathrm{S}_{1} ; S_{2} ; S_{3} ;:: ; S_{L}$ are encoded by $\mathrm{S}$ that is the transmission matrix of an STBC, where $[\mathrm{S}]_{i j}, i=1 ; 2 ;:: ; N_{t}$ and $j=1 ; 2 ;::: ; T$ represent element of the linear combination of symbols and their complex conjugates, which are transmitted simultaneously from the $i$-th transmit antenna in the $j$-th symbol periods [17]. S is independent identically distributed (i.i.d) Gaussian random signals with zero mean and variance matrix given by 


$$
E\left\{S_{n} S_{m} H\right\}=\left\{\begin{array}{l}
\sigma^{2}, n=m \\
0, n !=m
\end{array}\right.
$$

Where $E\{$.$\} implies the expectation and \sigma^{2}$ is the power accompanying one symbol. The input-output relation of the system can be written as

$$
\mathrm{Y}=\mathrm{HS}+\mathrm{W}
$$

Where $\mathrm{S}$ is the $N_{t} X T$ transmit matrix, $\mathrm{Y}$ is the $N_{r} X T$ received matrix and $\mathrm{W}$ is an $N_{r} X T$ i.i.d. Gaussian random noise matrix with zero mean. The MIMO channel response is described by $N_{r} x N_{t}$ matrix $\mathrm{H}$. A general entry of the channel matrix $\mathrm{H}$ is denoted by $\left\{h_{i j}\right\}$. This represents the complex gain of the channel between the $j$-th transmitter and the $i$-th receiver and can be written as

$$
\mathrm{H}=\left[\begin{array}{ccc}
h_{11} & \cdots & h_{1 n} \\
\vdots & \ddots & \vdots \\
h_{m 1} & \cdots & h_{m n}
\end{array}\right]
$$

Where

$$
\begin{aligned}
h_{i j} & =\alpha+j \beta=\sqrt{\alpha^{2}+\beta^{2}} \cdot \exp (j \arctan (\beta / \alpha)) \\
& =\left|h_{i j}\right| \cdot \operatorname{EXP}\left(j \Phi_{i j}\right)
\end{aligned}
$$

In a rich scattering environment with no line-of-sight (LOS), $\alpha$ and $\beta$ are independent and normal distributed random variables, then channel gains $\left\{h_{i j}\right\}$ are usually Rayleigh distributed.

The signals are transmitted over channel. The combined signal $\bar{r}_{n}$ at the receiver is

$\overline{\mathrm{r}}_{\mathrm{n}}=\left[c \sum_{i=1}^{n} \sum_{j=1}^{m}\left|h_{i j} \| h_{i j}\right|\right]+S n+W n=\mathrm{c}\|\mathrm{H}\|_{\mathrm{F}}^{2} \mathrm{~S}_{\mathrm{n}}+\mathrm{W}_{\mathrm{n}}, \mathrm{n}=1,2, \ldots, \mathrm{L}$

where $\mathrm{W}_{n}$ is the noise term after combining. At the receiver the maximum likelihood (ML) decoder is used to detect the transmitted symbol. The ML decoder can be simplified using the orthogonality of S. The received symbol, hence, can be determined by

$\dot{\mathrm{S}} \mathrm{n}=\arg \min \left|\overline{\mathrm{r}}_{\mathrm{n}}-\mathrm{c}\|\mathrm{H}\|_{\mathrm{F}}^{2} \mathrm{~S}\right|^{2}$

This is a low complexity orthogonal system model that can be considered highly attractive for practical applications.

\section{Channel Estimation Techniques}

Basically, methods of channel estimation can be classified according to four dimensions [17].

- $\quad$ From the view of estimation theory, there are

- Least Square (LS) Estimation

- Minimum Mean Square Error (MMSE) Estimation

- According to the processing domain, estimation can be done in 
- Time Domain

- Frequency Domain

- Due to the different pilot-symbol arrangements, there are

- Estimator with Block-Type Pilot (Training Based)

- Estimator with Comb-Type Pilot (Pilot Symbol Aided Modulation)

- With different estimation iteration, there are:

- Iterative Methods

- Direct Methods

\subsection{LS Estimation Technique}

The knowledge of CSI is required at the receiver to recover the transmitted signals properly in MIMO systems. In training based channel estimation, the training symbols that are known to the receiver are multiplexed along with the data stream and examined in the receiver to estimate the channel. In practice, training based LS estimation is more frequently used due to its acceptable performance. But this estimation involves matrix inversions, which result in high computational complexity and hence undesirable for hardware implementation. The orthogonal matrix Triangularization is a very convenient technique to avoid matrix inversion and is preferable because of its clever implementation in highly parallel array architecture.

In aNtXNrMIMO system, totally ( $N t X N r$ ) channels are needed to be estimated between transmitters and receivers. The received training symbols can be expressed as

$$
\mathrm{y}=\mathrm{Hx}+\mathrm{n}
$$

where $\mathrm{x}$ is the transmitted training signal, $\mathrm{y}$ is the received signal and $\mathrm{n}$ is the noise response. The channel response $\mathrm{H}$ is assumed to be random and quasi-static within two transmission blocks. The LS approach solves the estimation equation (4.1) by minimizing the cost function as,

$$
J(\mathrm{H})=(\mathrm{y}-\mathrm{Hx})^{H}(\mathrm{y}-\mathrm{Hx})
$$

The gradient of equation (4.2) is given below,

$$
\frac{\partial}{\partial H} J(\mathrm{H})=-2 \mathrm{x}^{H} \mathrm{y}+2 \mathrm{x}^{H} \mathrm{x}
$$

Minimizing the gradient to zero yields the LS estimation $\hat{H}$ of the channel response obtained by

$$
\hat{\mathrm{H}}=\left(\mathrm{x}^{H} \mathrm{x}\right)^{-1} \mathrm{x}^{H} \mathrm{y}
$$

\subsection{MMSE Technique}

Improved performance compared to ZF can be obtained by attempting to minimize both interference and noise at the same time. This is done by the Minimum Mean Squared Error (MMSE) detection scheme, which is a trade off between noise inflation and interference suppression. The weight matrix for MMSE detection is given by:

$$
W^{H}=\mathrm{H}^{\mathrm{H}}\left(\rho^{2} I_{m}+H H^{\mathrm{H}}\right)^{\mathrm{H}}
$$

Where $\mathrm{I}_{\mathrm{M}}$ and $\mathrm{I}_{\mathrm{N}}$ are the $\mathrm{Mx} \mathrm{M}$ and $\mathrm{N} \times \mathrm{N}$ identity matrices respectively. 


\subsection{Proposed Channel Estimation Technique}

The inversion of $x^{H} \mathrm{x}$ in equation (4.4) has a high complexity and will significantly increase when the size of $\mathrm{x}$ increases, which is dependent on the number of transmit antennas. To avoid complexity because of matrix inversion, orthogonal matrix triangularization is applied on $\mathrm{x}$. The matrix triangularization can be calculated via Householder transformation, or Givens rotation. The Givens rotation is a recursive method that requires a larger number of floating point operations as compared to the Householder transformation method [19]. In this work, Householder transformation is chosen to minimize the required operations. In the Householder approach, a series of reflection matrix is applied to the matrix, $\mathrm{x}$, column by column to annihilate the lower triangular elements. The reflection transformations are orthonormal matrices that can be written as

$$
\mathrm{A}=\left(\mathrm{I}+\beta \mathrm{v} \mathrm{v}^{H}\right)
$$

where $\mathrm{v}$ is the Householder vector and $\beta=-2\|\mathrm{v}\|^{2}$. For the matrix $\mathrm{x}$, to annihilate the lower elements of the $k$-th column the $\mathrm{A}_{k}$ is constructed as follows:
i. Let $\mathrm{v}$ equal the $k$-th column of $\mathrm{x}$
ii. Update $\mathrm{v}$ by $\mathrm{v}=\mathrm{x}+\|\mathrm{x}\|^{2} \Upsilon$, where $\Upsilon=[1 ; 0 ;: \therefore ; 0]^{T}$
iii. Determine $\beta$ by $\beta=-2\|v\|_{2}^{2}$
iv. $\mathrm{A}_{k}$ is calculated according to equation (4.6).

The $\mathrm{A}_{k}$ formed from the above steps are pre-multiplied by $\mathrm{x}$ sequentially as follows

$$
\mathrm{A} n, \ldots \ldots \ldots . . . ., \mathrm{A} 1 \mathrm{x}=\left[\begin{array}{l}
R \\
0
\end{array}\right]
$$

where, $\mathrm{R}$ is a $n x n$ upper triangular matrix, 0 is a null matrix, and the sequence of re ${ }^{\circ}$ ection matrices form the complex transpose of the orthogonal matrix $\mathrm{Q}^{H}$, i.e., $\mathrm{Q}^{H}=\mathrm{A} n$, $\mathrm{A} 1$ and $\mathrm{I}=\mathrm{Q}^{H} \mathrm{Q}$.

Thus, equation (4.7) can be written as

$$
\mathrm{x}=\mathrm{Q}\left[\begin{array}{l}
R \\
0
\end{array}\right]
$$

The error function for estimation in equation (4.4) can be expressed as

$$
\varepsilon=\mathrm{y}-\dot{H} \mathrm{x} ; \text { if } \varepsilon=0 \text {; then } \mathrm{y}=\dot{\mathrm{H}} \mathrm{x}
$$

By combining equations (4.6) and (4.7) the received signal stands

$$
\mathrm{y}=\dot{\mathrm{H}} \mathrm{x}=\dot{\mathrm{HQ}}\left[\begin{array}{l}
R \\
0
\end{array}\right]
$$

The Hermitian of $\mathrm{Q}\left[\begin{array}{l}R \\ 0\end{array}\right]$ is multiplied to both sides of equation (4.10) to derive the proposed channel estimation

$$
\dot{\mathrm{H}}_{O M T}=\mathrm{yQ}^{H}\left[\begin{array}{c}
R \\
0
\end{array}\right] H
$$


As $\mathrm{R}$ is an upper triangular matrix, $\dot{\mathrm{H}}_{O M T}$ can be solved through back-substitution. The proposed estimation is a numerically stable low complexity solution to channel estimation of MIMO systems.

\section{Result and Discussion}

This section presents comparisons between Block error rates (BER) for the different channel estimation schemes. The results presented here are obtained from simulations performed in MATLAB. The channel is considered to be Rayleigh and the modulation scheme to be BPSK for simplicity in simulation. The block error rate is calculated by performing $10^{\wedge} 6$ trials at each SNR point. The block error rate is calculated for each SNR value. Performing $10^{\wedge} 6$ trials at each SNR value refers to sending same block of data $10^{\wedge} 6$ times from transmitter towards receiver for that SNR value. If the transmitted block of data don't match with the detected data at the receiver then an error occurs. I calculate the total number of error that has occurred during $10^{\wedge} 6$ trials. The block error rate is then calculated for a SNR value as total number of error divided by total number of trials. As an example if total number of error is $5 \times 10^{\wedge} 5$ for $10^{\wedge} 6$ trials at a SNR value then the block error rate for this SNR value is

\section{Block error rate $=500000 / 1000000=1 / 2$}

A new realization of channel was chosen in each trial and for each SNR value.

The simulation results shown in this section are limited to 4 by 4 and 2 by 2 transmit and receive antenna MIMO system only. The BER performance of the different channel estimation techniques and AlamoutiSTBC can be analysed under varying number of antennas.

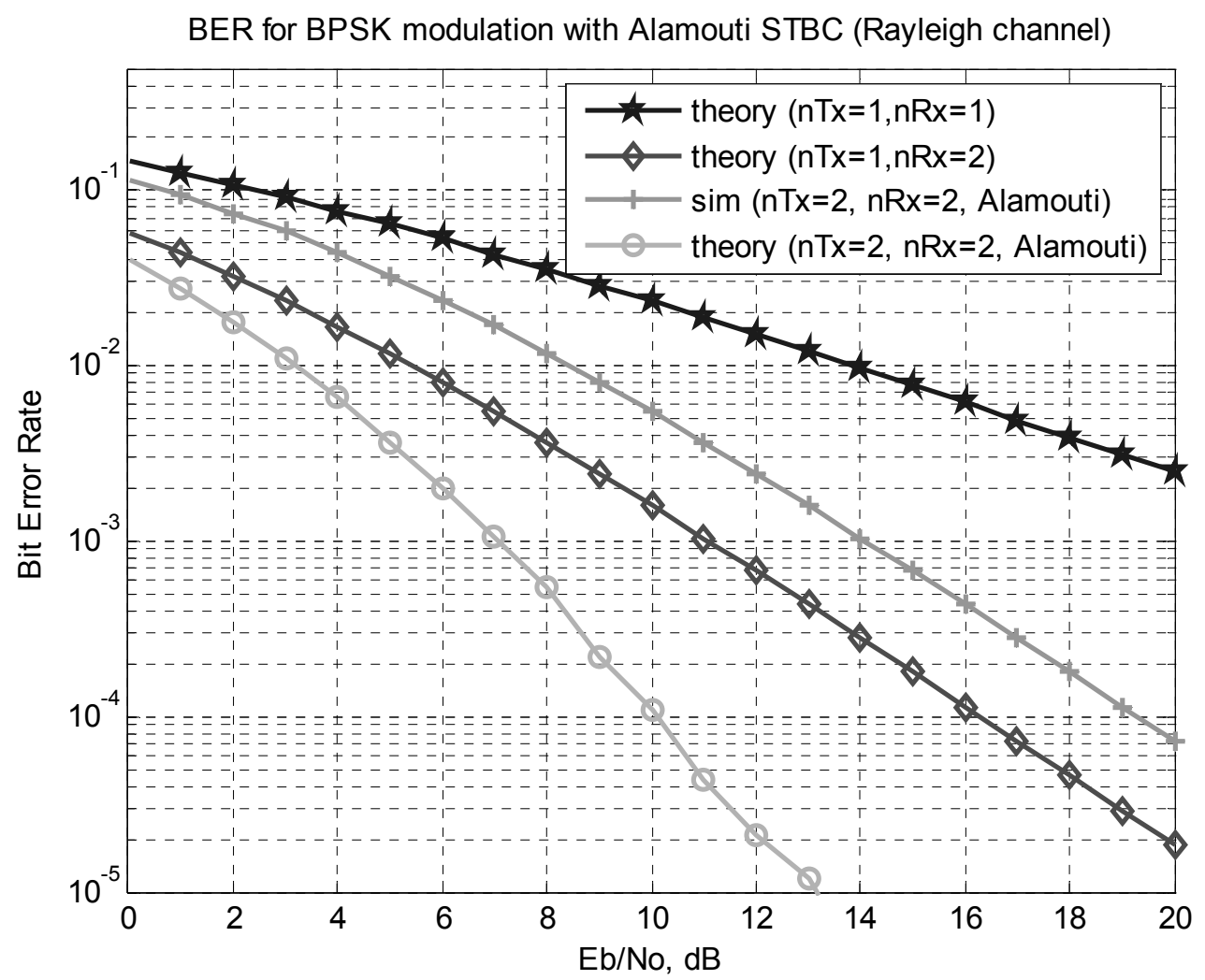

Fig 5.1 BER of Alamouti's STBC with different number of antenna 
The BER performance of the MIMO system using AlamoutiSTBC is shown in the figure 5.1 for varying number of antennas. The BER is plotted for up to 2 transmit and 2 receive antenna. It can be seen from above figure that as the number of transmit antenna increase, the BER performance is also increasing and the same for increasing number of receive antenna. This figure also shows the BER obtained from theoretical formula. As a fact, the simulated BER is lower than that obtained theoretically. The value of BER for simulated AlamoutiSTBC is nearly equal to $10^{-2}$ at SNR equal to $8 \mathrm{~dB}$ for 2 transmit and 2 receive antenna whereas it is nearly equal to $0.02 \times 10^{-3}$ for same SNR.

Figure 5.2 and figure 5.3 show the simulated results of BER in dB for LS and MMSE channel estimation techniques in MIMO with 4 transmit and 4 receive antennas against $\mathrm{SNR}$ in $\mathrm{dB}$, respectively..

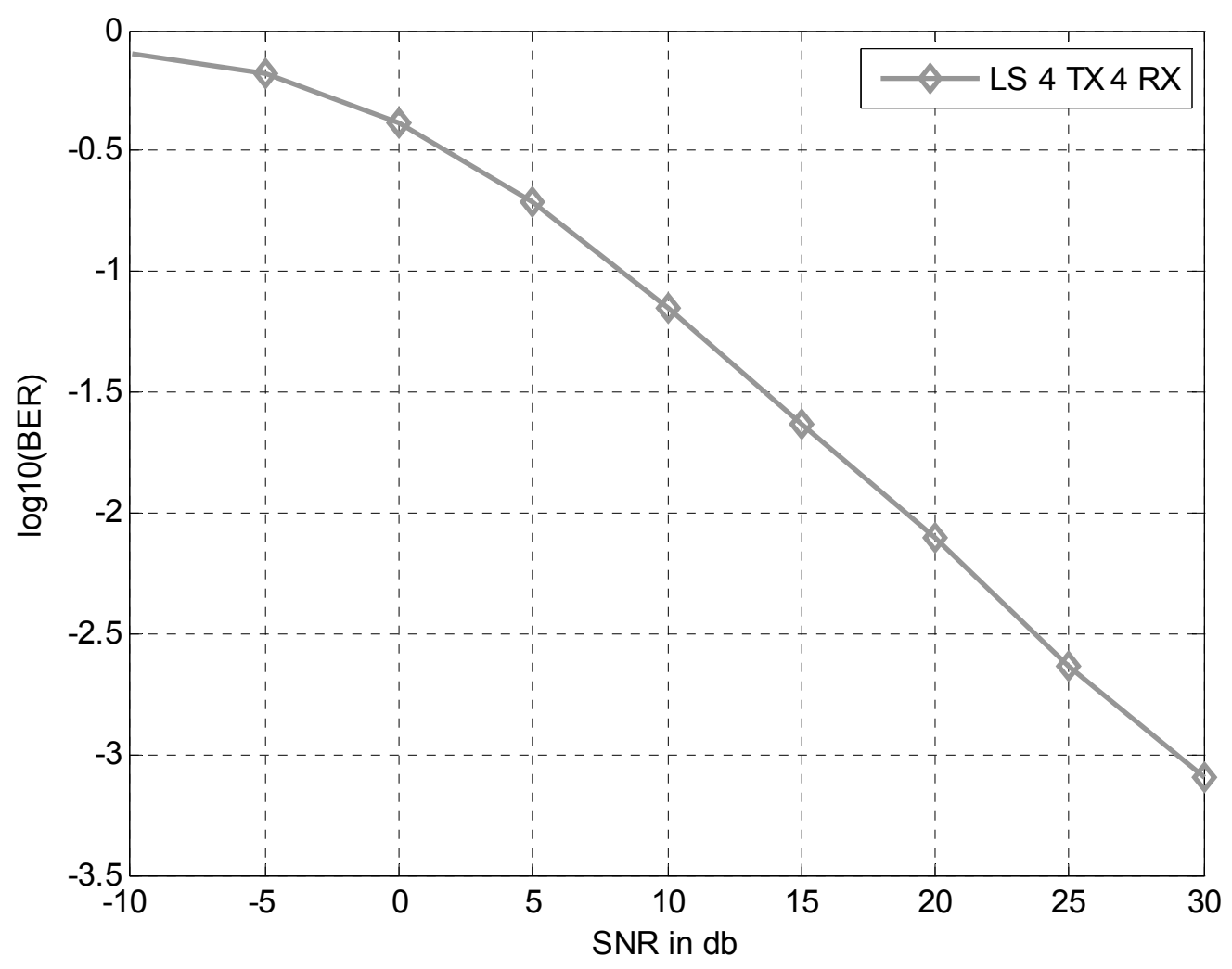

Fig 5.2 BER performance of LS scheme 


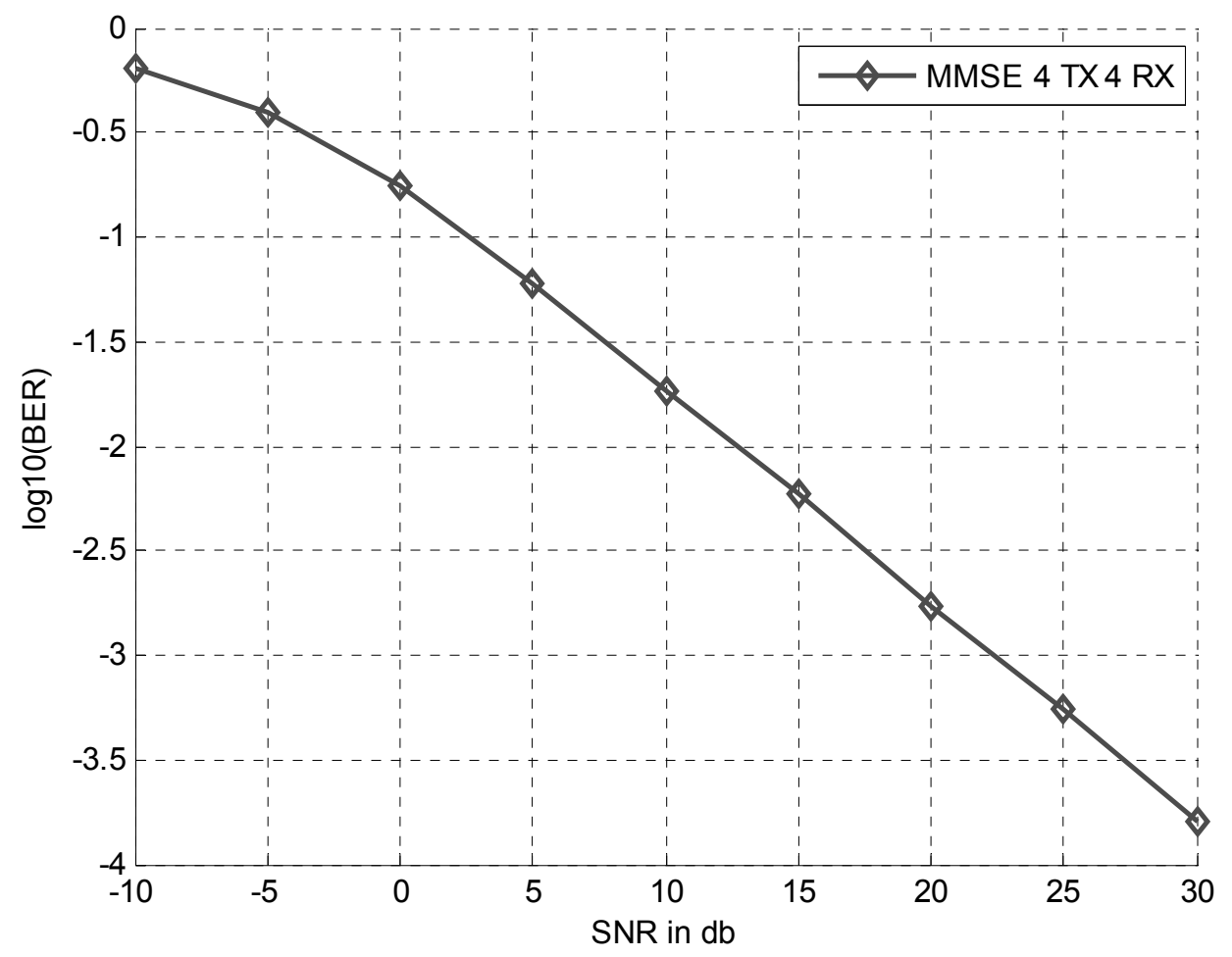

Fig 5.3 BER performance of MMSE scheme

The value of BER in LS and MMSE schemes are equal to $-0.1 \mathrm{~dB}$ and $-0.3 \mathrm{~dB}$ at $\mathrm{SNR}=-10 \mathrm{~dB}$, respectively. As the value of SNR goes on increasing, the value of the BER also goes on increasing. Finally, the value of BER is equal to $-3.6 \mathrm{~dB}$ and -3.9 at $\mathrm{SNR}=30 \mathrm{~dB}$. It is clear that increasing SNR gives lower value of BER resulting better BER performance and MMSE channel estimation technique provides better BER performance than LS scheme.

To get better view of LS and MMSE schemes, these two schemes are simulates under the same environment with only 2 transmit and 2 receive antenna due to memory insufficiency problem and the simulated results are shown in figure 5.4. Again, it can be seen that MMSE scheme provides better BER performance than LS scheme. The value of BER is about $0.5 \times 10^{-1} \& 0.25 \times 10^{-1} \mathrm{~dB}$ at average $\mathrm{E}_{\mathrm{b}} / \mathrm{N}_{0}=0 \mathrm{~dB}$ and $0.5 \times 10^{-3} \& 10^{-5} \mathrm{~dB}$ at $\mathrm{E}_{\mathrm{b}} / \mathrm{N}_{0}=20 \mathrm{~dB}$, respectively. 
BER for BPSK modulation for 2x2 MIMO with LS \& MMSE (Rayleigh channel)

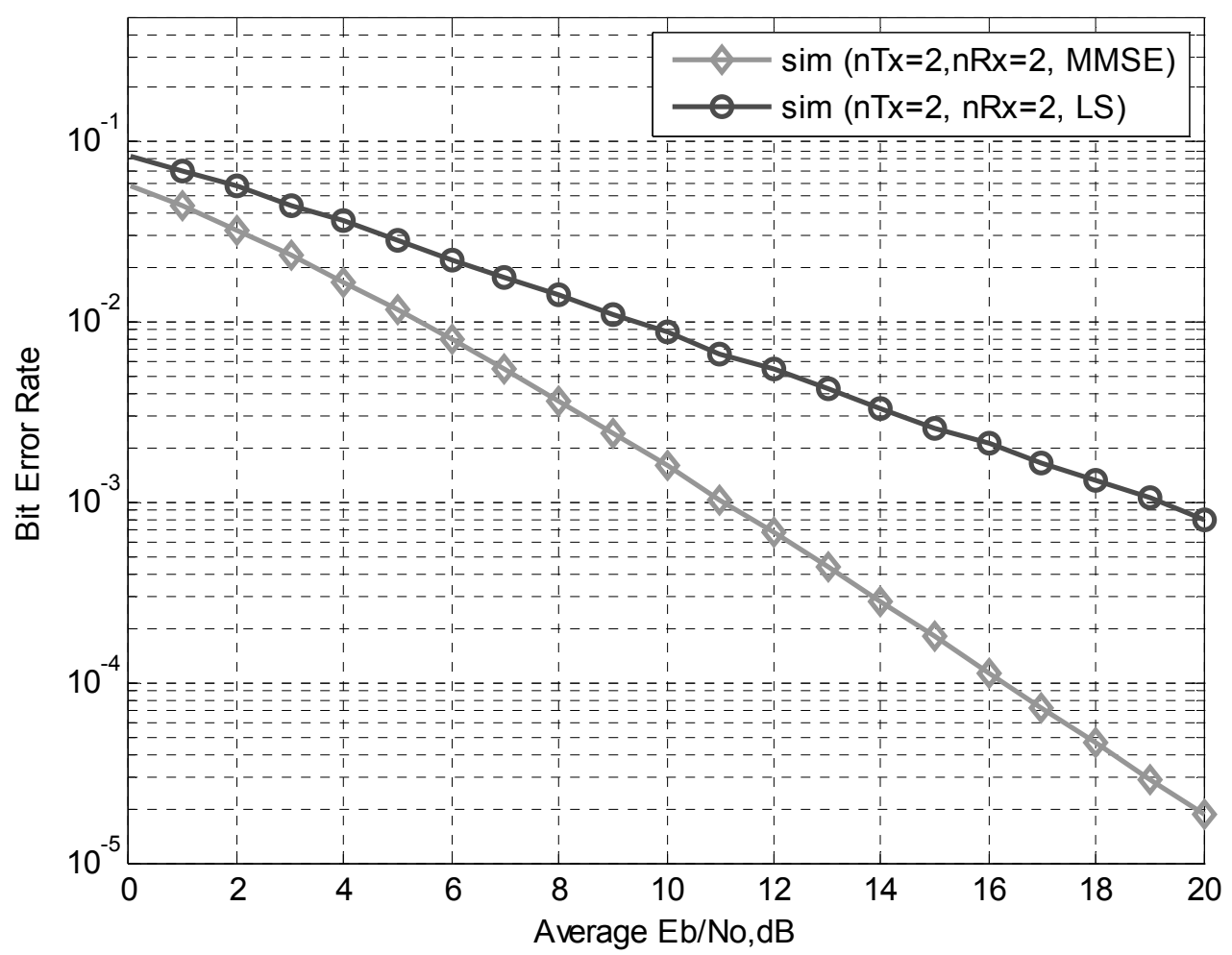

Figure 5.4 Comparison of BER performance of LS and MMSE schemes

The BER values for modified (proposed) LS channel estimation technique along with LS and MMSE techniques are simulated and plotted in figure 5.5 for 2 transmit and 2 receive antenna against different values of SNR ranging from 0 to $20 \mathrm{~dB}$. 
BER for BPSK modulation for 2x2 MIMO with LS \& MMSE \& Modified LS (Rayleigh channel)

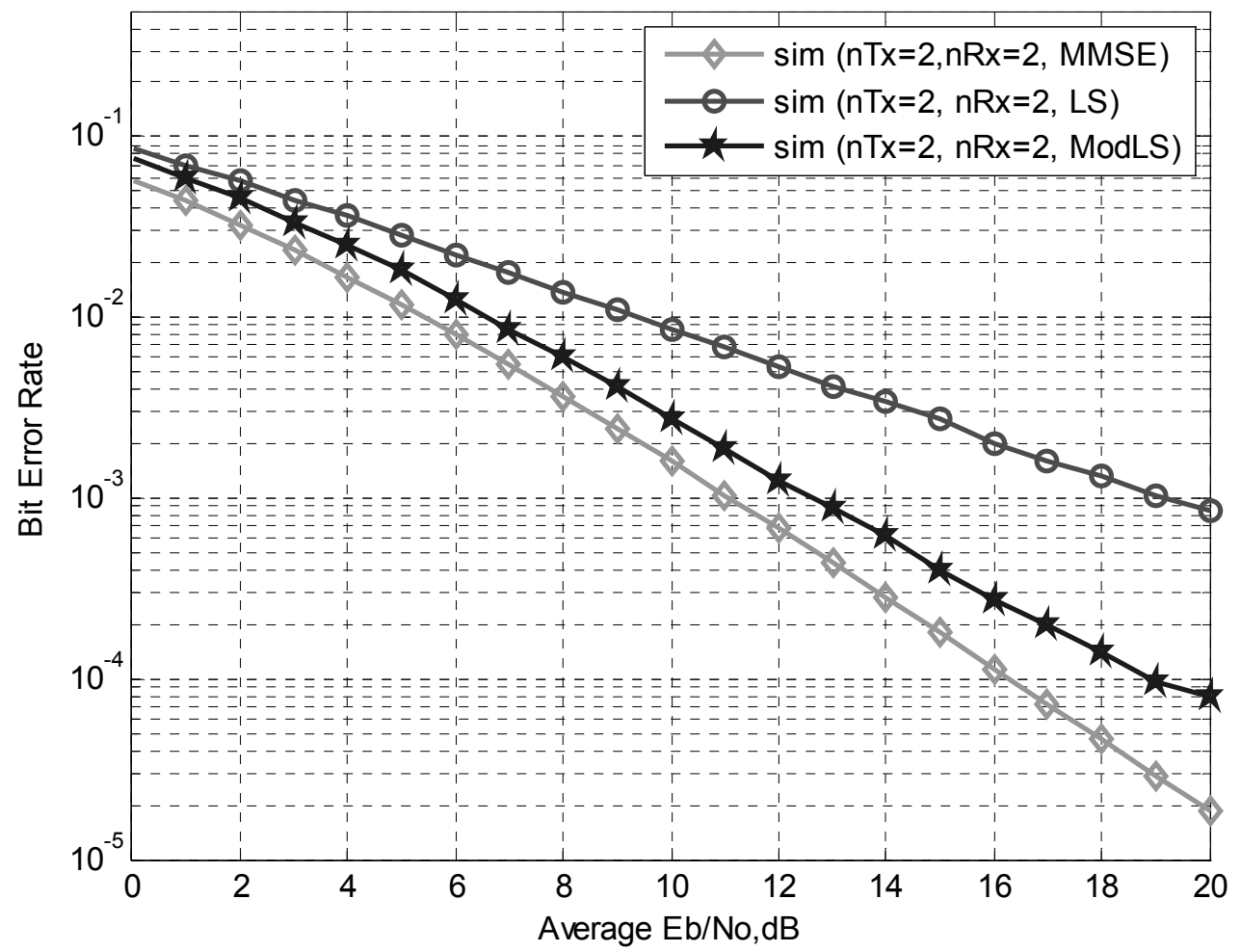

Fig 5.5 Comparison of BER Performance of Modified LS with LS and MMSE schemes

As seen from results in figure 5.5, the modified LS scheme, as expected, offers significant improvement in terms of BER performance of the channel estimation than conventional LS scheme. The value of BER is almost same at lower value of SNR, but at higher SNR as $20 \mathrm{~dB}$, the modified LS scheme provides BER of about 10-4 dB instead of 10-3 dB in LS scheme. In addition, the MMSE scheme has the least values of BER than LS and modified LS schemes.

Finally, computational complexity of modified LS scheme against LS and MMSE schemes is carried out in terms of number of floating - point calculations with respect to varying number of the transmit antenna and simulated results are shown in the figure 5.6. The computational complexity is increasing with increasing the number of transmit antennas for all of these three schemes but with different rates. The rate of increase of complexity in modified LS scheme is almost linear whereas rising exponential in LS and MMSE schemes. Thus, complexity of modified LS scheme is, as expected, lower than that of LS scheme as well as MMSE scheme. In addition, the computational complexity is almost same for these channel estimation schemes up to 3 transmit antenna but then varies with higher number of transmit antennas. 


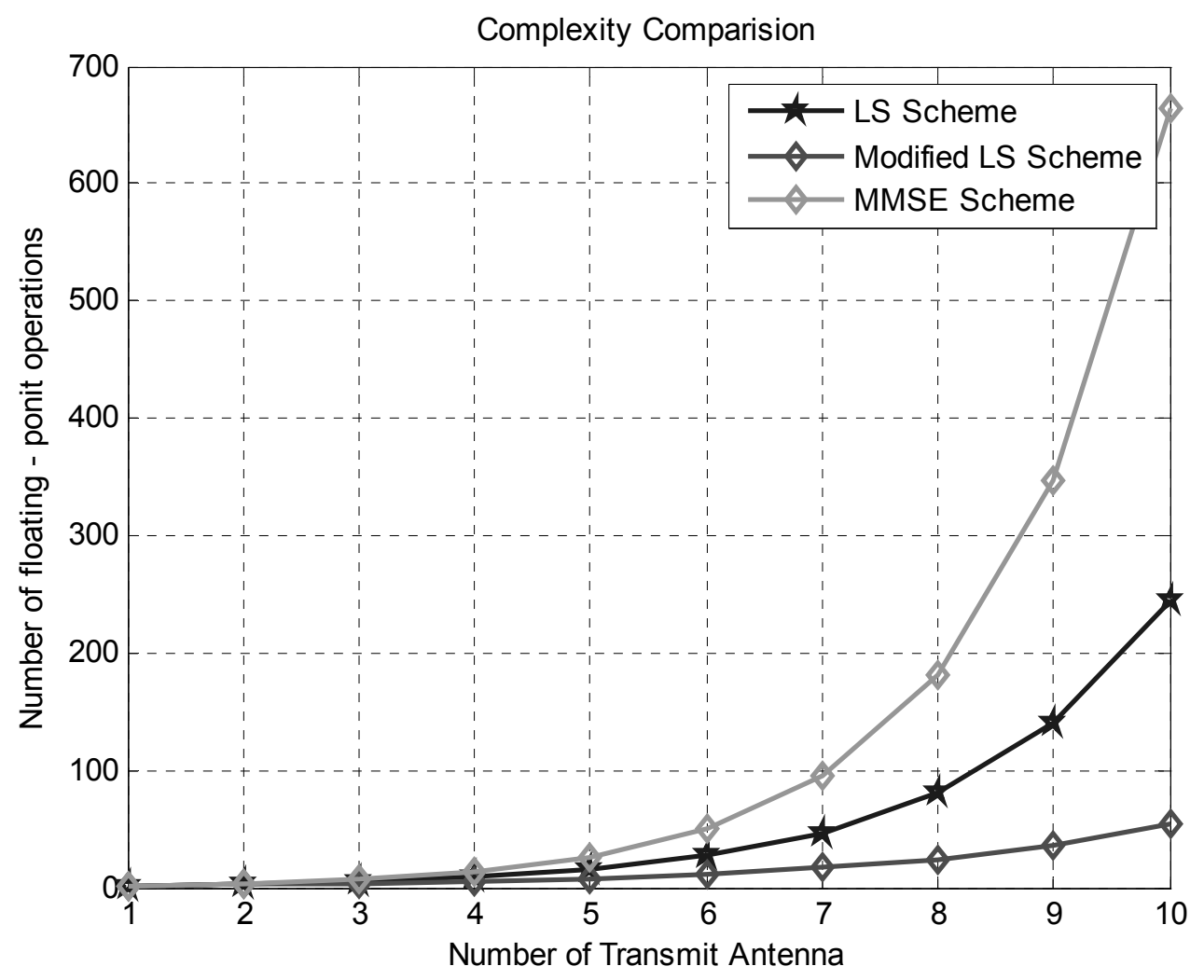

Fig 5.6 Complexity comparison between LS, MMSE and modified LS schemes

\section{Conclusion}

The simulation results for BER and computational complexity have been presented and discussed in the last chapter 5. From the results, it can be concluded that MMSE scheme is superior to LS and modified LS schemes in terms of BER performance and modified LS scheme is superior to LS and MMSE schemes in terms of the computational complexity.

The MMSE channel estimation technique can be chosen when very good BER performance is required in MIMO system at the cost of higher computational complexity and the LS channel estimation can be used with lesser complexity but with poor BER performance. Hence, modified LS scheme serves midway between these two schemes and can be chosen for good BER performance still having least computational complexity.

Thus, this paper, as expected, shows that modified (proposed) LS scheme offers less computational complexity but better BER performance than the LS scheme. 


\section{References}

1. A. A. Abouda, and S. G. HÄaggman, "Effect of mutual coupling on capacity of MIMO wireless channels in high SNR scenario”, Progress In Electromagnetic Research, PIER 65, 27-40, 2006.

2. A. A. Abouda, H. M. El-Sallabi, and S. G. H.Äaggman, "Effect of antenna array geometry and ULA azimuthal orientation on MIMO channel properties in urban city street grid", Progress In Electromagnetic Research, PIER 64, 257-278, 2006.

3. J. Paulraj, D. A. Gore, R. U. Nabar, and H. Bolcskei, "An overview of MIMO communications A key to gigabit wireless", Proceedings of the IEEE, Vol. 92, No. 2, 198-18, Feb. 2004.

4. R. Chen, H. Zhang, Y. Xu, and H. Luo, "On MM-type channel estimation for MIMOOFDM systems”, IEEE Trans. Wireless Commun., Vol. 6, No. 3, 1046-1055, May 2007.

5. Van De Beek, O. Edfors, M. Sandell, S. K. Wilson, and P. O. BÄorjesson, "On channel estimation in OFDM systems", Proc. IEEE Int. Veh. Technol. Conf., Chicago, Jul. 1995.

6. G. H. Golub, and C. F. Van Loan, "Matrix Computations", $3^{\text {rd }}$ edition, John Hopkins University Press, Maryland, 1996.

7. D. WÄubben, J. Rinas, R. BÄohnke, V. KÄuhn, and K. D. Kammeyer, "Efficient algorithm for decoding layered space-time codes”, Electron. Lett., Vol. 37, No. 22, 1348-1350, Oct. 2001.

8. V. Pham, M. Le, L. Mai, and G. Yoon, "Low complexity maximum-likelihood decoder for VBLAST-STBC scheme in MIMO wireless communication systems", IEEE63rd Vehicular Technology Conference, 2006, Vol. 5, 2309-2313, May 2006.

9. Z. Guo, Y. Wang, L. Li, X. Tao, P. Zhang, and H. Harada, "A hybrid detection algorithm for MIMO systems", International Conference on Communications, Circuits and Systems Proceedings 2006, Vol. 2, 883-887, Jun. 2006.

10. H. Dhungana, "Performance Evaluation of MIMO - OFDM System by PAPR Reduction and Channel Estimation”, Thesis No. 066/MSI/607, Pulchowk Campus, IOE, TU, November 2011

11. S. K. Sharma, "MIMO - OFDM Techniques for Wireless Communication System”, Thesis No. IC 64615, Pulchowk Campus, IOE, TU, October 2010

12. K. P. Bagadi, Prof. Das, S., "MIMO - OFDM Channel Estimation using Pilot Carriers " Jan, 2013

13. S. W. Patil, Prof. A. N. Jadhav., "Channel Estimation using LS and MMSE Estimators", Jun, 2013

14. Beak, Edfors, Sandell, Wilson, Borjesson, “On Channel Estimation in OFDM System ”, Jan, 2012

15. T. S. Rappport, “Wireless Communication: Principle and Practice”, Second Edition, Pearson, 2013

16. F. Delestre, "Channel Estimation and Performance Analysis of MIMO - OFDM using SpaceTime and Space - Frequency Coding Schemes", Jun 2011

17. W. Zhang, "Channel and Frequency Offset Estimation for OFDM - Based System", Jun, 2010

18. Qi Wang, "Study of Channel Estimation in MIMO-OFDM Systems for Software Defined Radio", LITH-ISY-EX--07/3954—SE, Linköping 2007

19. S. M. Alamouti, "A simple transmit diversity technique for wireless communications", IEEE J. Select. Areas Commun., Vol. 16, 1451 \{1458, Oct. 1998. 